\title{
ON TOPOLOGICAL COMPLETENESS ${ }^{1}$
}

\section{W. COHEN}

Recently A. Weil ${ }^{2}$ defined a uniform space as a set of points $p$ such that for each $\alpha$ in a set $A$ there is defined a set $U_{\alpha}(p) \subset S$, the class of sets $U_{\alpha}(p)$ satisfying the conditions:

$\mathrm{I}_{\mathbf{A}} \cdot \prod_{\alpha} U_{\alpha}(p)=(p)$.

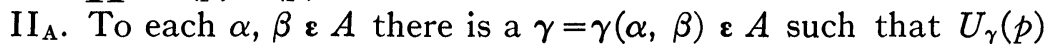
$\subset U_{\alpha}(p) U_{\beta}(p)$.

III $_{\mathrm{A}}$. To each $\alpha \varepsilon A$ there is a $\beta(\alpha) \varepsilon A$ such that if $p^{\prime}, p^{\prime \prime} \varepsilon U_{\beta(\alpha)}(q)$, then $p^{\prime \prime} \varepsilon U_{\alpha}\left(p^{\prime}\right)$.

For the uniform space $S$, Weil introduced the concept of Cauchy family $\left\{M_{\beta}\right\}$ of sets. Such a family is defined by the conditions that the intersection of any finite number of sets of the family is nonempty and that to each $\alpha \varepsilon A$ there is a $p_{\alpha} \varepsilon S$ and a $\beta(\alpha)$ such that $M_{\beta(\alpha)} \subset U_{\alpha}\left(p_{\alpha}\right)$. Weil gives a theory of completeness in these terms.

The writer has considered ${ }^{3}$ a space $S$ of points $p$ and neighborhoods $U_{\alpha}(p)$ where $\alpha$ is an element of a set $A$ such that:

I. $\prod_{\alpha} U_{\alpha}(p)=(p)$.

II. To each $\alpha$ and $\beta \varepsilon A$ and $p \varepsilon S$ there is a $\gamma=\gamma(\alpha, \beta ; p)$ such that $U_{\gamma}(p) \subset U_{\alpha}(p) U_{\beta}(p)$.

III. To each $\alpha \varepsilon A$ and $p \varepsilon S$ there are $\lambda(\alpha), \delta(p, \alpha) \varepsilon A$ such that, if $U_{\delta(p, \alpha)}(q) U_{\lambda(\alpha)}(p) \neq 0$, then $U_{\delta(p, \alpha)}(q) \subset U_{\alpha}(p)$.

The uniformity conditions here are lighter than those in $\mathrm{II}_{\mathrm{A}}$ and III $_{\mathrm{A}}$. A Cauchy sequence $p_{n} \varepsilon S$ was defined by the condition that for every $\alpha \varepsilon A, n_{\alpha}$ and $p_{\alpha} \varepsilon S$ exist such that $p_{n} \varepsilon U_{\alpha}\left(p_{\alpha}\right)$ for $n \geqq n_{\alpha} . S$ is complete if every Cauchy sequence has a limit. It was shown that there is a complete space $S^{*}$ which contains a homeomorphic image of $S$ such that the image of a Cauchy sequence in $S$ is a convergent sequence in $S^{*}$.

It is the object of this paper to show that Weil's space is a special case of the space $S_{I, I I, I I I}$ and that the notion of Cauchy family in this space leads to the same theory of completeness as that previously developed.

THEOREM 1. If $S$ satisfies III $_{\mathrm{A}}$ and $\beta^{2}(\alpha)=\beta(\beta(\alpha))$, then from $U_{\beta^{2}(\alpha)}(q) U_{\beta^{2}(\alpha)}(p) \neq 0$ follows $U_{\beta^{2}(\alpha)}(q) \subset U_{\alpha}(p)$.

${ }^{1}$ Presented to the Society, December 27, 1939.

2 A. Weil, Sur les Espaces a Structure Uniforme, Paris, 1938.

${ }^{3} \mathrm{~L}$. W. Cohen, On imbedding a space in a complete space, Duke Mathematical Journal, vol. 5 (1939), pp. 174-183. Also Duke Mathematical Journal, vol. 3 (1937), pp. 610-617, where the notion of topological completeness is introduced. 
Proof. Let $s \varepsilon U_{\beta^{2}(\alpha)}(q) U_{\beta^{2}(\alpha)}(p)$. Then from $s, q^{\prime} \varepsilon U_{\beta^{2}(\alpha)}(q)$ we have $q^{\prime} \varepsilon U_{\beta(\alpha)}(s)$. Therefore $U_{\beta^{2}(\alpha)}(q) \subset U_{\beta(\alpha)}(s)$. Similarly $U_{\beta^{2}(\alpha)}(p)$ $\subset U_{\beta(\alpha)}(s)$. Now from $p \varepsilon U_{\beta(\alpha)}(s)$ and $U_{\beta^{2}(\alpha)}(q) \subset U_{\beta(\alpha)}(s)$ we have $U_{\beta^{2}(\alpha)}(q) \subset U_{\alpha}(p)$.

Corollary. If $S$ satisfies III $_{\mathrm{A}}$, then $S$ satisfies III.

Proof. For any $p \varepsilon S$ and $\alpha \varepsilon A$ we need only take $\lambda(\alpha)=\delta(p, \alpha)$ $=\beta(\beta(\alpha))$. The result is stronger than III since $\delta(p, \alpha)$ is independent of $p$.

From now on a space $S$ is one satisfying I, II, III. A family of sets $\left\{M_{\beta}\right\}$ is a Cauchy family if the intersection of any finite number of $M_{\beta}$ is non-empty and if for any $\alpha \varepsilon A$ there is a $\beta(\alpha)$ such that $M_{\beta(\alpha)} \subset U_{\alpha}\left(p_{\alpha}\right)$ for some $p_{\alpha} \varepsilon S$. We will say that $S$ is $W$-complete if, for every Cauchy family $\left\{M_{\beta}\right\}, \prod_{\beta} \bar{M}_{\beta} \neq 0$, where $\bar{M}_{\beta}$ is the closure of $M_{\beta}$. We shall always use the notations $\lambda(\alpha), \delta(p, \alpha)$ in the sense of III.

Theorem 2. $S$ is $W$-complete if and only if every Cauchy family $\left\{U_{\alpha}\left(p_{\alpha}\right)\right\}$ consisting of one $U_{\alpha}\left(p_{\alpha}\right)$ for each $\alpha \varepsilon A$ has the property that $\prod_{\alpha} U_{\alpha}\left(p_{\lambda(\alpha)}\right) \neq 0$.

Proof. Assume $S$ is $W$-complete. If $\left\{U_{\alpha}\left(p_{\alpha}\right)\right\}$ is a Cauchy family, then $\prod_{\alpha} \bar{U}_{\lambda(\alpha)}\left(p_{\lambda(\alpha)}\right) \neq 0$. Since $\bar{U}_{\lambda(\alpha)}(p) \subset U_{\alpha}(p)$ follows from III, the condition $\prod_{\alpha} U_{\alpha}\left(p_{\lambda(\alpha)}\right) \neq 0$ is necessary. Assume now that the condition is satisfied and let $\left\{M_{\beta}\right\}$ be a Cauchy family in $S$. To each $\alpha \varepsilon A$ there are $p_{\alpha} \varepsilon S$ and $M_{\beta(\alpha)}$ such that $M_{\beta(\alpha)} \subset U_{\alpha}\left(p_{\alpha}\right)$. It is clear that $\left\{U_{\alpha}\left(p_{\alpha}\right)\right\}$ is a Cauchy family. Hence there is $p \varepsilon \prod_{\alpha} U_{\alpha}\left(p_{\lambda(\alpha)}\right)$. For any $\gamma \varepsilon A$, consider the $\lambda(\gamma)$ and $\alpha=\delta(p, \gamma)$ of III. Since $p \varepsilon U_{\alpha}\left(p_{\lambda(\alpha)}\right) U_{\lambda(\gamma)}(p), U_{\alpha}\left(p_{\lambda(\alpha)}\right) \subset U_{\gamma}(p)$ and

$$
M_{\beta(\lambda(\alpha))} \subset U_{\lambda(\alpha)}\left(p_{\lambda(\alpha)}\right) \subset U_{\alpha}\left(p_{\lambda(\alpha)}\right) \subset U_{\gamma}(p) .
$$

Hence

$$
0 \neq M_{\beta} M_{\beta(\lambda(\alpha))} \subset M_{\beta} U_{\gamma}(p)
$$

for all $\beta, \gamma$. Thus $p \varepsilon \prod_{\beta} \bar{M}_{\beta}$ and $S$ is $W$-complete.

The space $S^{*}$ referred to above is defined as follows. A family $\left\{U_{\alpha}\left(p_{\alpha}\right)\right\}$, one for each $\alpha \varepsilon A$, such that for any $\left(\alpha_{1}, \cdots, \alpha_{n}\right) \subset A$, $U_{\alpha_{1}}\left(p_{\alpha_{1}}\right) U_{\alpha_{2}}\left(p_{\alpha_{2}}\right) \cdots U_{\alpha_{n}}\left(p_{\alpha_{n}}\right) \neq 0$ is denoted by $\Pi$. We write $\Pi^{\prime} \sim \Pi^{\prime \prime}$ if for every $\alpha \varepsilon A$ there is a set $\left(\alpha_{1}, \cdots, \alpha_{n}\right) \subset A$ such that for some $p_{\alpha} \varepsilon S$

$$
\prod_{i=1}^{n} U_{\alpha_{i}}\left(p_{\alpha_{i}}^{\prime}\right)+\prod_{i=1}^{n} U_{\alpha_{i}}\left(p_{\alpha_{i}}^{\prime \prime}\right) \subset U_{\alpha}\left(p_{\alpha}\right)
$$


The relation $\Pi^{\prime} \sim \Pi^{\prime \prime}$ classifies all $\Pi$ in to mutually exclusive classes $C(\Pi)$ which are the points $P$ of $S^{*}$. The neighborhoods $U_{\alpha_{1}}^{*} \ldots \alpha_{n}(P)$ are made up of all $Q=C\left(\Pi^{Q}\right) \varepsilon S^{*}$ where $\Pi^{Q}=\left\{U_{\alpha}\left(q_{\alpha}\right)\right\}$ is such that for some $\beta_{i}=\beta_{i}\left(\alpha_{1}, \cdots, \alpha_{n} ; P\right), i=1, \cdots, m=m\left(\alpha_{1}, \cdots, \alpha_{n} ; P\right)$,

$$
\prod_{i=1}^{m} U_{\beta_{i}}\left(q_{\beta_{\imath}}\right) \subset \prod_{i=1}^{n} U_{\alpha_{i}}\left(p_{\alpha_{i}}\right) .
$$

The space $S^{*}$ (for which $A^{*}$ is the set of all finite subsets of $A$ ) satisfies I, II and

III*. For each $\left(\alpha_{1}, \cdots, \alpha_{n}\right) \subset A$ and for $P \varepsilon S^{*}$ there exist $\gamma_{i}=\gamma_{i}\left(\alpha_{1}, \cdots, \alpha_{n} ; P\right), i=1, \cdots, m=m\left(\alpha_{1}, \cdots, \alpha_{n} ; P\right)$, such that if $U_{\gamma_{1}}^{*} \cdots \gamma_{m}(Q) U_{\gamma_{1}}^{*} \cdots \gamma_{m}(P) \neq 0$, then $U_{\gamma_{1}}^{*} \cdots \gamma_{m}(Q) \subset U_{\alpha_{1}}^{*} \cdots \alpha_{n}(P)$.

The mapping $f(p)=C\left(\Pi^{p}\right)$, where $\Pi^{p}=\left\{U_{\alpha}(p)\right\}$, is the homeomorphism on $S$ to a subset of $S^{*}$ referred to above.

Theorem 3. $S^{*}$ is $W$-complete.

Proof. We first show that if $\left\{U_{\alpha_{1}}^{*} \cdots \alpha_{n}\left(P^{\alpha_{1}} \cdots \alpha_{n}\right)\right\}$, where each $\left(\alpha_{1}, \cdots, \alpha_{n}\right) \subset A$ occurs just once, is a Cauchy family in $S^{*}$, then

$$
\prod_{\left(a_{1} \cdots \alpha_{n}\right) \subset A} \overline{U_{\alpha_{1}}^{*} \cdots \alpha_{n}}\left(p^{\alpha_{1} \cdots \alpha_{n}}\right) \neq 0 .
$$

Consider the $U_{\alpha}^{*}\left(P^{\alpha}\right)$ for all $\alpha \varepsilon A$. Now $P^{\alpha}=C\left(\Pi^{P^{\alpha}}\right), \Pi^{P^{\alpha}}=\left\{U_{\gamma}\left(p_{\gamma}^{\alpha}\right)\right\}$, $U_{\gamma}\left(p_{\gamma}^{\alpha}\right)$ being a neighborhood in $S$ for each $(\alpha, \gamma) \subset A$. Since, for each $\left(\alpha_{1}, \cdots, \alpha_{n}\right) \subset A, \prod_{i=1}^{n} U_{\alpha_{i}}^{*}\left(P^{\alpha_{i}}\right) \neq 0$, we have

$$
\prod_{i=1}^{n} U_{\alpha_{i}}\left(p_{\alpha_{i}}^{\alpha_{i}}\right) \neq 0 .
$$

Hence the family $\Pi=\left\{U_{\alpha}\left(p_{\alpha}^{\alpha}\right)\right\}$ defines a $P=C(\Pi) \varepsilon S^{*}$. For any $\left(\gamma_{1}, \cdots, \gamma_{m}\right) \subset A$

$$
\prod_{i=1}^{m} U_{\gamma_{i}}^{*}(P)=U_{\gamma_{1} \cdots \gamma_{m}}^{*}(P) .
$$

Both $U_{\gamma_{i}}^{*}(P)$ and $U_{\gamma_{i}}^{*}\left(P^{\gamma_{i}}\right)$ are the set of all $Q=C\left(\Pi^{Q}\right), \Pi^{Q}=\left\{U_{\beta}\left(q_{\beta}\right)\right\}$ such that for some $\beta_{j i}=\beta_{j}\left(\gamma_{i}\right), j=1, \cdots, k_{i}, \prod_{j=1}^{k_{i}} U_{\beta_{j i}}\left(q_{\beta_{j i}}\right) \subset U_{\gamma_{i}}\left(p_{\gamma_{i}}^{\gamma_{i}}\right)$. Hence

$$
U_{\gamma_{i}}^{*}(P)=U_{\gamma_{i}}^{*}\left(P^{\gamma_{i}}\right), \quad U_{\gamma_{1} \cdots \gamma_{m}}^{*}(P)=\prod_{i=1}^{m} U_{\gamma_{i}}^{*}\left(P^{\gamma_{i}}\right) .
$$

Thus from the fact that $\left\{U_{\alpha_{1}}^{*} \cdots \alpha_{n}\left(P^{\alpha_{1}} \cdots \alpha_{n}\right)\right\}$ is a Cauchy family we have for any sets $\left(\alpha_{1}, \cdots, \alpha_{n}\right),\left(\gamma_{1}, \cdots, \gamma_{m}\right) \subset A$ 


$$
U_{\alpha_{1} \cdots \alpha_{n}}^{*}\left(P^{\alpha_{1} \cdots \alpha_{n}}\right) U_{\gamma_{1} \cdots \gamma_{m}}^{*}(P)=U_{\alpha_{1} \cdots \alpha_{n}}^{*}\left(P^{\alpha_{1} \cdots \alpha_{n}}\right) \prod_{i=1}^{m} U_{\gamma_{i}}^{*}\left(P^{\gamma_{i}}\right) \neq 0 .
$$

It follows that

$$
P \varepsilon \prod_{\left(\alpha_{1} \cdots \alpha_{n}\right) \subset_{A}} \overline{U_{\alpha_{1}}^{*} \cdots \alpha_{n}}\left(P^{\alpha_{1} \cdots \alpha_{n}}\right) \text {. }
$$

Now suppose that $\left\{M_{\beta}^{*}\right\}$ is a Cauchy family of sets in $S^{*}$. For every $\left(\gamma_{1}, \cdots, \gamma_{n}\right) \subset A$ there is a $\beta\left(\gamma_{1}, \cdots, \gamma_{n}\right)$ such that for some $P_{\gamma_{1}} \cdots \gamma_{n} \varepsilon S^{*}, M_{\beta}^{*}\left(\gamma_{1} \cdots \gamma_{n}\right) \subset U_{\gamma_{1} \cdots \gamma_{n}}^{*}\left(P^{\gamma_{1} \cdots \gamma_{n}}\right)$. Thus

$$
0 \neq \prod_{i=1}^{m} M_{\beta\left(\gamma_{1}, \cdots, \gamma_{n_{i}}\right)}^{*} \subset \prod_{i=1}^{m} U_{\gamma_{1}, \cdots, \gamma_{n_{i}}^{2}}^{*}\left(P^{\gamma_{1}^{i}}, \cdots, \gamma_{n_{i}}^{i}\right)
$$

for every finite set $\left(\gamma_{1}^{\imath}, \cdots, \gamma_{n_{i}}^{\imath}\right) \subset A$ and $\left\{U_{\gamma_{1}}^{*} \cdots_{\gamma_{n}}\left(P_{\gamma_{1}} \cdots \gamma_{n}\right)\right\}$ is a Cauchy family. Let $P \varepsilon S^{*}$ satisfy (1). For any $\left(\alpha_{1}, \cdots, \alpha_{n}\right) \subset A$ and $P \varepsilon S^{*}$ let $\left(\gamma_{1}, \cdots, \gamma_{m}\right) \subset A$ be the set of $\gamma_{i}=\gamma_{i}\left(\alpha_{1}, \cdots, \alpha_{n} ; P\right)$ satisfying III*. From III* and (1)

$$
U_{\gamma_{1} \cdots \gamma_{m}}^{*}\left(P^{\gamma_{1} \cdots \gamma_{m}}\right) \subset U_{\alpha_{1} \cdots \alpha_{n}}^{*}(P) .
$$

Since $\left\{M_{\beta}^{*}\right\}$ is a Cauchy family, for any $\beta$ and $\left(\alpha_{1}, \cdots, \alpha_{n}\right) \subset A$ we have

$$
\begin{gathered}
0 \neq M_{\beta}^{*} M_{\beta\left(\gamma_{1}, \cdots, \gamma_{m}\right)}^{*} \subset M_{\beta\left(\gamma_{1}, \cdots, \gamma_{m}\right)}^{*} \subset U_{\gamma_{1} \cdots \gamma_{m}}^{*}\left(P_{\gamma_{1}} \cdots \gamma_{m}\right) \subset U_{\alpha_{1} \cdots \alpha_{n}}^{*}(P), \\
M_{\beta}^{*} U_{\alpha_{1} \cdots \alpha_{n}}^{*}(P) \neq 0 .
\end{gathered}
$$

Hence $P_{\varepsilon} \prod_{\beta} \overline{M_{\beta}^{*}}$ and $S^{*}$ is $W$-complete.

Theorem 4. If $\left\{M_{\beta}\right\}$ is a Cauchy family in $S$ and $f(S) \subset S^{*}$ is the homeomorphism defined above, then there is a $P \varepsilon S^{*}$ such that

1. $\prod_{\beta} \overline{f\left(M_{\beta}\right)}=(P)$,

2. for any $\left(\alpha_{1}, \cdots, \alpha_{n}\right) \subset A$ there are $\beta_{1}, \cdots, \beta_{m}$ such that

$$
\prod_{i=1}^{m} f\left(M_{\beta_{i}}\right) \subset U_{\alpha_{1} \cdots \alpha_{n}}^{*}(P) \text {. }
$$

Proof. $f\left(M_{\beta}\right)$ is the class of $P=C\left(\Pi^{p}\right)$ for all $p \varepsilon M_{\beta}$ where for any $\left(\alpha_{1}, \cdots, \alpha_{n}\right) \subset A$ and $\lambda\left(\alpha_{i}\right)$ there are $\beta\left(\lambda\left(\alpha_{i}\right)\right)$ and $p_{\lambda\left(\alpha_{i}\right)}$ such that

$$
\begin{gathered}
M_{\beta\left(\lambda\left(\alpha_{i}\right)\right)} \subset U_{\lambda\left(\alpha_{i}\right)}\left(p_{\lambda\left(\alpha_{i}\right)}\right) \subset U_{\alpha_{i}}\left(p_{\lambda\left(\alpha_{i}\right)}\right), \\
0 \neq \prod_{i=1}^{n} M_{\beta\left(\lambda\left(\alpha_{i}\right)\right)} \subset \prod_{i=1}^{n} U_{\alpha_{i}}\left(p_{\lambda\left(\alpha_{i}\right)}\right) .
\end{gathered}
$$

If $q \varepsilon \prod_{i=1}^{n} M_{\beta\left(\lambda\left(\alpha_{i}\right)\right)}$, then for $\delta_{i}=\delta\left(p_{\lambda\left(\alpha_{i}\right)}, \alpha_{i}\right)$ we have

$$
q \varepsilon U_{\delta_{i}}(q) U_{\lambda\left(\alpha_{i}\right)}\left(p_{\lambda\left(\alpha_{i}\right)}\right), \quad U_{\delta_{i}}(q) \subset U_{\alpha_{i}}\left(p_{\lambda\left(\alpha_{i}\right)}\right), \quad i=1,2, \cdots, n .
$$


Since

$$
q \varepsilon \prod_{i=1}^{n} U_{\delta_{i}}(q) \subset \prod_{i=1}^{n} U_{\alpha_{i}}\left(p_{\lambda\left(\alpha_{i}\right)}\right),
$$

the family $\left\{U_{\alpha}\left(p_{\lambda(\alpha)}\right)\right\}=\Pi$ defines a $P=C(\Pi) \varepsilon S^{*}$ such that

$$
f(q) \varepsilon U_{\delta_{1} \cdots \delta_{n}}^{*}(f(q)) \subset U_{a_{1} \cdots \alpha_{n}}^{*}(P) .
$$

Hence for such $\left(\alpha_{1}, \cdots, \alpha_{n}\right) \subset A$ there are $P^{\alpha_{1} \cdots \alpha_{n}}=P$ and $\beta_{i}=\beta\left(\lambda\left(\alpha_{i}\right)\right), i=1, \cdots, n$, such that

$$
0 \neq \prod_{i=1}^{n} f\left(M_{\left.\beta\left(\lambda\left(\alpha_{i}\right)\right)\right)} \subset U_{\alpha_{1}}^{*} \ldots \alpha_{n}(P) .\right.
$$

Thus the family of all finite products $\left\{f\left(M_{\beta_{1}}\right) \cdots f\left(M_{\beta_{n}}\right)\right\}$ is a Cauchy family in $S^{*}$ since $f\left(M_{\beta_{1}}\right) \cdots f\left(M_{\beta_{n}}\right)=f\left(M_{\beta_{1}} \cdots M_{\beta_{n}}\right) \neq 0$. We have

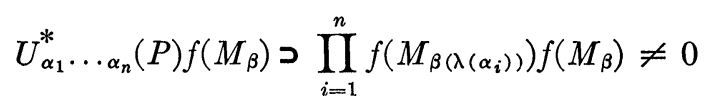

for all $\beta$ and all $\left(\alpha_{1}, \cdots, \alpha_{n}\right) \subset A$. In other words

$$
P \varepsilon \prod_{\beta} \overline{f\left(M_{\beta}\right)} .
$$

But the intersection of all $\overline{f\left(M_{\beta}\right)}$ is $P$, for if $P^{\prime}$ is any other point there are sets $\left(\alpha_{1}, \cdots, \alpha_{n}\right),\left(\alpha_{1}^{\prime}, \cdots, \alpha_{n^{\prime}}^{\prime}\right) \subset A$ such that

and

$$
U_{\alpha_{1} \cdots \alpha_{n}}^{*,}(P) U_{\alpha_{1} \cdots \alpha_{n}{ }^{\prime}}^{* \prime}\left(P^{\prime}\right)=0
$$

$$
P^{\prime} \varepsilon S^{*}-\overline{U_{\alpha_{1}}^{*} \cdots \alpha_{n}}(P) \subset S^{*}-\prod_{i=1}^{n} \overline{f\left(M_{\beta\left(\lambda\left(\alpha_{i}\right)\right)}\right.} \subset S^{*}-\prod_{\beta} \overline{f\left(M_{\beta}\right)} .
$$

We conclude with the remark that if $S$ is $W$-complete, $S$ is complete. Suppose $p_{n}$ is a Cauchy sequence in $S$. Let $M_{n}=\left(p_{n}, p_{n+1}, \cdots\right)$. Then the intersection of any finite set of $M_{n}$ is non-empty and for any $\alpha \varepsilon A$ there is an $n(\alpha)$ such that $M_{n(\alpha)} \subset U_{\alpha}\left(p_{\alpha}\right)$ for some $p_{\alpha} \varepsilon S$. Thus $\left\{M_{n}\right\}$ is a Cauchy family. $S$ being $W$-complete, there is a $p \varepsilon \prod_{n} \bar{M}_{n}$. Now for $p$, any $\alpha \varepsilon A, \lambda(\alpha)$ and $\delta=\delta(p, \alpha)$, we have

$$
M_{n(\delta)} \subset U_{\delta}\left(p_{\delta}\right), \quad 0 \neq U_{\lambda(\alpha)}(p) M_{n(\delta)} \subset U_{\lambda(\alpha)}(p) U_{\delta}\left(p_{\delta}\right),
$$

$M_{n(\delta)} \subset U_{\delta}\left(p_{\delta}\right) \subset U_{\alpha}(p), p_{n} \varepsilon U_{\alpha}(p), n \geqq n(\delta)=n(\delta(p, \alpha)), \lim p_{n}=p$.

UNIVERSITY OF KentuCKY 